\title{
A Quantum Differential Evolutionary Algorithm for the Independent Set Problem
}

\author{
Omar Kettani, Faycal Ramdani, Benaissa Tadili \\ LPG Lab. \\ Scientific Institute \\ Mohamed V University, Rabat
}

\begin{abstract}
The Independent Set problem consists to find a maximum cardinality subset of vertices of a given graph such that no two vertices are adjacent. In this paper, we propose a quantum evolutionary algorithm which uses a differential operator to update the quantum angles of the superposition state of Q-bits for solving this problem. Simulation results on some graph examples show that this approach is effective.
\end{abstract}

\section{General Terms}

Graph theory, Stochastic Algorithm.

\section{Keywords}

Independent Set, Quantum evolutionary algorithm.

\section{INTRODUCTION}

In graph theory, the Maximum Independent Set is a maximum cardinality subset of vertices in which no two vertices are adjacent. This problem which has many applications in computer science is computationally NP-hard in general [1], but can be solved in polynomial time on some special graph classes [2] [3]. For large and hard instances of this problem, approximation algorithms and heuristic approaches are required to obtain near optimal solutions within reasonable amount of computation time.

Quantum computing, based on the concepts and principles of quantum theory, such as superposition of quantum states, entanglement and intervention, was first proposed by Benioff [4]. It was successfully applied later to many optimization and combinatorial problems [5] [6] [7].

Recently, an evolutionary algorithm was proposed for tackling the Independent Set Problem [8] and another approach based on ellipsoids was presented in [9] in order to provide exact solutions to this problem. In this work, we propose a quantum evolutionary algorithm using a differential operator to update the quantum angles of the superposition state of Q-bits for solving this problem.

Given an undirected graph $\mathrm{G}=(\mathrm{V}, \mathrm{E})$ with vertex set $\mathrm{V}=\mathrm{V}$ (G) of cardinality $|\mathrm{V}(\mathrm{G})|=\mathrm{n}$, and edge set $\mathrm{E}=\mathrm{E}(\mathrm{G})$ of cardinality $|\mathrm{E}(\mathrm{G})|=\mathrm{m}$.

The neighborhood of a vertex $v \in V$ is the set $N(v)=\{u \in V$ : vu $\in \mathrm{E}\}$.

A set $\mathrm{S} \subseteq \mathrm{V}(\mathrm{G})$ is independent if no two vertices from $\mathrm{S}$ are adjacent; by $\operatorname{Ind}(G)$ we mean the set of all the independent sets of $\mathrm{G}$.

This paper is organized as follows: In section II we describe the proposed approach for solving this problem, section III provides some examples of the application of the proposed algorithm. Finally, concluding remarks are given in section IV.

II Quantum Evolutionary Algorithm is inspired from the concepts of quantum computing, where the smallest unit of information is called Q-bit and is defined as $[\alpha, \beta]^{\mathrm{T}}$, where $\alpha$ and $\beta$ are complex numbers that specify the probability amplitude of the respective Q-bit states such that $|\alpha|^{2}+|\beta|^{2}$ $=1 .|\alpha|^{2}$ is the probability that the Q-bit will be in state ' 0 ' and $|\beta|^{2}$ represents the probability that the Q-bit will be in state ' 1 '.

The representation for an individual $\mathrm{q}$ in the population with $\mathrm{m}$-bit is given as follows:

$$
\begin{aligned}
& \alpha_{1}, \ldots, \alpha_{\mathrm{m}} \\
& \mathrm{q}=\left(\begin{array}{l}
\mid \\
\beta_{1}, \ldots, \beta_{\mathrm{m}}
\end{array}\right. \\
& \text { where }\left|\alpha_{\mathrm{i}}\right|^{2}+\left|\beta_{\mathrm{i}}\right|^{2}=1, \mathrm{i}=1,2, \ldots \mathrm{m} . \\
& \text { and } \theta_{\mathrm{i}}=\arctan \left(\left|\beta_{\mathrm{i}}\right| /\left|\alpha_{\mathrm{i}}\right|\right) \text { is the phase of the ith qubit. }
\end{aligned}
$$

\section{Description of the algorithm:}

First, the population is initialized with the $\alpha_{\mathrm{i}}$ and $\beta_{\mathrm{i}}$ of all bits of all individuals set to $1 / \sqrt{ } 2$. At each generation, the binary strings are generated from the respective Q-bit strings by observing the Q-bit states using the following rule:

$P_{i, j}=1$, if rand $(0,1)<\sin \left(\theta_{i, j}\right)^{2}$ and $P_{i, j}=0$ else. (1)

where $P_{i, j}$ is represents the $j$ th bit of ith individual in the population and $\theta_{i, j}$ its phase.

The fitness value of the population strings generated is evaluated and the best solutions are stored separately.

The mutant Q-bits $\theta_{\mathrm{i}}{ }^{\mathrm{m}}$ are generated for all the individuals in the population in every generation.

Mutant Q-bits of the $\mathrm{i}$ individual in generation $\mathrm{t}$ are determined as follows:

$\theta_{\mathrm{i}}^{\mathrm{mt}}=\theta_{\mathrm{r} 1}+\mathrm{F}^{\mathrm{t}} \cdot\left(\theta_{\mathrm{r} 2}-\theta_{\mathrm{r} 3}\right)$

where $r_{1}, r_{2}, r_{3}$ and $i$ are mutually distinct and $F^{t}$ is the mutation control parameter which is determined in every generation by

$\mathrm{F}^{\mathrm{t}}=\operatorname{rand}(0,1)$

where rand is a random number generated from a uniform distribution on $[0,1]$

The crossover operation transform the original Q-bits and the respective mutant Q-bits by using the following rule: 


$$
\theta_{i, j}{ }^{c t}=\left\{\begin{array}{l}
\theta_{i, j}{ }^{m t} \text { if }\left(\operatorname{rand}_{i}(0,1) \leq \mathrm{CR}\right) \text { or }\left(\mathrm{i}=\mathrm{I}_{\text {rand }}\right) \\
\theta_{\mathrm{i}, \mathrm{j}}{ }^{\mathrm{t}} \text { if }\left(\operatorname{rand}_{\mathrm{i}}(0,1)>\mathrm{CR}\right) \text { and }\left(\mathrm{i} \neq \mathrm{I}_{\text {rand }}\right)
\end{array}\right.
$$

where $\theta_{\mathrm{i}, \mathrm{j}}{ }^{\mathrm{ct}}$ is the ith Q-bit of jth individuals after the crossover operation. $\mathrm{I}_{\mathrm{rand}}$ is an integer randomly chosen from $\{1,2, \ldots \mathrm{n}\}$ which ensures that at least one Q-bit is different from the original set in each individual. $\mathrm{CR}=0.5$, is the control parameter which is found to be the best value experimentally.

The population and the Q-bits are updated by observing the state of the newly obtained Q-bits $\theta_{\mathrm{i}, \mathrm{j}}{ }^{\mathrm{ct}}$, a new set of individuals are generated which replace the corresponding individual in the population if their fitness values are higher. The replacement is done by using the following rules:

$$
P_{j}^{t+1}= \begin{cases}P_{j}^{c t} & \text { if } f\left(P_{j}^{c t}\right)>f\left(P_{j}^{t}\right) \\ P_{j}^{t} & \text { otherwise }\end{cases}
$$

and

$$
\theta_{i, j}{ }^{t+1}=\left\{\begin{array}{l}
\theta_{i, j}{ }^{c t} \text { if } f\left(P_{j}^{c t}\right)>f\left(P_{j}{ }^{t}\right) \\
\theta_{i, j}{ }^{t} \text { otherwise }
\end{array}\right.
$$

where $P_{j}^{c}$ is the $j$ th individual by observing the Q-bits modified after crossover $\left(\theta_{i, j}{ }^{\text {ct }}\right)$ and $f\left(P_{j}\right)$ is the fitness value of the corresponding individual.

According to [10], we can choose the fitness function $f$ to maximize, as follows :

$$
f(x)=e^{T} x-x^{T} A_{G} x / 2
$$

where $\mathrm{A}_{\mathrm{G}}$ is the adjacency matrix of the input graph and $\mathrm{x} \in\{0,1\}^{\mathrm{n}}$ is the indicator vector of a subset of vertices of $\mathrm{G}$ and $\mathrm{e}^{\mathrm{T}}=(1, \ldots, 1)$.

We summarize the pseudo code of the proposed algorithm as follows:

input: $A_{G}$, the adjacency matrix of graph $G$

output: an independent set $\mathrm{S}$

initialize $\mathrm{Q}(\mathrm{t})$;

make $\mathrm{P}(\mathrm{t})$ from $\mathrm{Q}(\mathrm{t})$ by using $(1)$

$\mathrm{t} \leftarrow 1$

$\mathrm{CR} \leftarrow 0.5$

evaluate the fitness function $\mathrm{f}$ of $\mathrm{P}(\mathrm{t})$ by using (5)

while $\mathrm{t}<\mathrm{T}$ do

$$
\mathrm{t} \leftarrow \mathrm{t}+1
$$

$\mathrm{F}^{\mathrm{t}} \leftarrow$ rand $(0,1)$

apply mutation on $\mathrm{Q}(\mathrm{t})$ by using (2)

make $\mathrm{Q}^{\mathrm{C}}(\mathrm{t})$ by crossover by using (3)

make $\mathrm{P}^{\mathrm{C}}(\mathrm{t})$ from $\mathrm{Q}^{\mathrm{C}}(\mathrm{t})$ by using (1)

evaluate fitness of $\mathrm{P}(\mathrm{t})$

update $\mathrm{P}(\mathrm{t}+1)$ and $\mathrm{Q}(\mathrm{t}+1)$ by (4) end while

output $\mathrm{P}=\operatorname{ArgMax}(\mathrm{f})$ as the indicator vector of the independent set $\mathrm{S}$.

\section{Examples}

In order to assess the performance of this method, tests have been conducted on some graph examples taken from the literature. The test environment is P4 3G, Windows 7, Matlab [11].

The population sizes was set to 20 . The number of generations was set to 100 . The obtained results are reported in the following table:

\begin{tabular}{|l|l|lllll|l|}
\hline Graph & n & \multicolumn{7}{|l|}{ independent } & set & Elapsed time ( s.) \\
\hline found & & & & & \\
\hline Octahedron & 6 & 1 & 4 & & & & 8.89 \\
\hline Cube & 8 & 2 & 4 & 6 & 8 & & 10.57 \\
\hline Grötzsch & 11 & 2 & 3 & 4 & 5 & 6 & 18.32 \\
\hline Herschel & 11 & 2 & 4 & 5 & 7 & 9 & 18.006 \\
11 & & & & & \\
\hline Icosahedron & 12 & 3 & 5 & 7 & & 31.99 \\
\hline Wheel & 8 & 3 & 5 & 7 & & 21.65 \\
\hline Petersen & 10 & 3 & 5 & 7 & 8 & & 24.97 \\
\hline $\begin{array}{l}\text { Ramsey } \\
\text { graph R(4,4) }\end{array}$ & 17 & 4 & 11 & 16 & & 58.63 \\
\hline
\end{tabular}

Table 1. The independent set found by the proposed method on the tested graph examples.

In the following figures where tested graphs are presented, white vertex belongs to the independent set found by the proposed method.

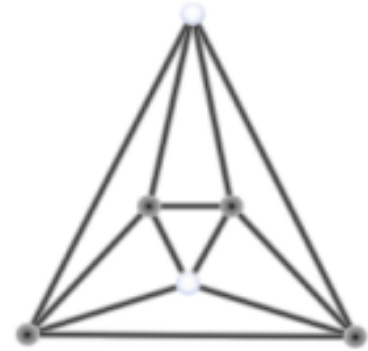

Figure 1: The Octahedron 


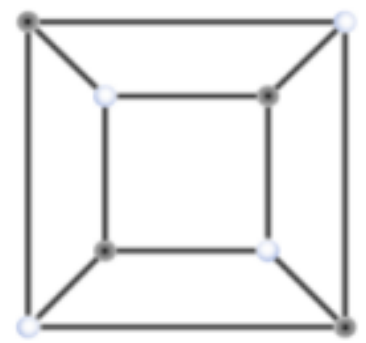

Figure 2: The Cube

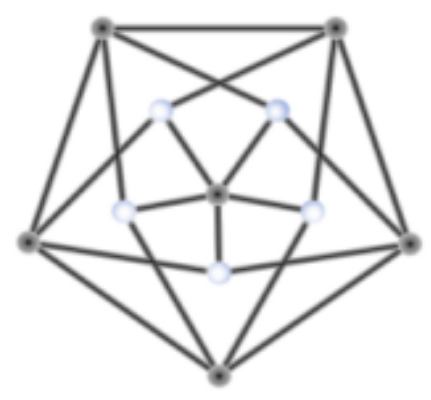

Figure 3: The Grötzsch graph [12]

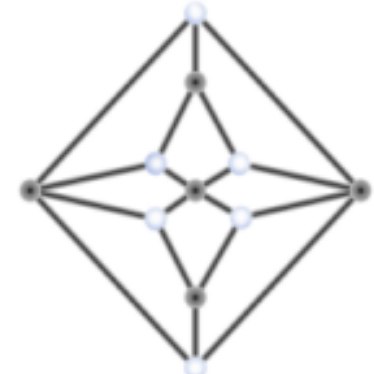

Figure 4: The Herschel graph [13]

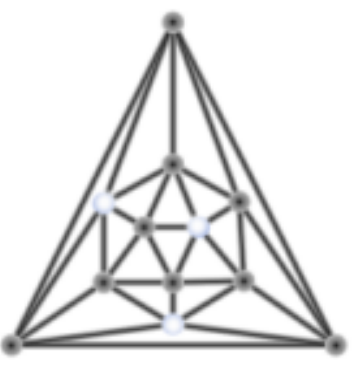

Figure 5: The Icosahedron

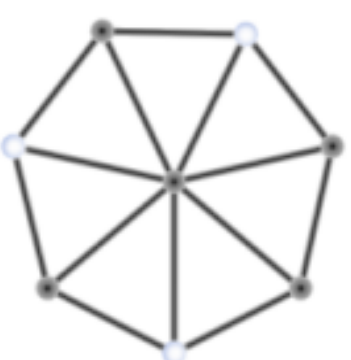

Figure 6: The Wheel graph W8

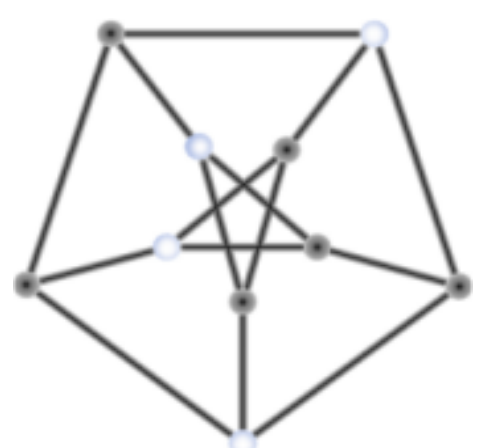

Figure 7: The Petersen graph [14]

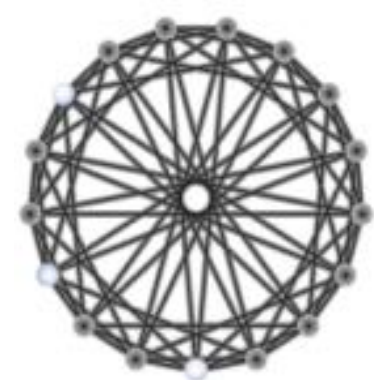

Figure 8: The Ramsey graph [15]

\section{CONCLUSIONS}

In this paper, we have described a quantum differential evolutionary algorithm in attempt to solve the Independent Set problem. We have tested this method on some graph examples. Simulation results show the effectiveness of the proposed approach. Further research will be concerned with the improvement of this method. To enhance its performance in both speed and quality, it would be interesting to find some adequate initialization phase instead of random initialization and to test this approach on large graph instances.

\section{REFERENCES}

[1] M. R. Garey, D. S. Johnson, Computers and Intractability: A Guide to the theory NP-completeness, San Francisco:Freeman (1979).

[2] R. Mosca, "Polynomial algorithms for the maximum stable set problem on particular classes of P5-free graphs,"Information Processing Letters,61, 1997, pp.137143. 
[3] A. Brandstädt, C.T. Hoàng, V.B. Le, "Stability number of bull-and chair-free graphs revisited," Discrete Appl. Math. 131 (2003) 39-50.

[4] P. Benioff, The computer as a physical system: a microscopic quantum mechanical hamiltonian model of computers as represented by Turing machines, J. Stat. Phys. 22 (1980) 563-591.

[5] B. Jiao, X. Gu and G. Xu, "An Improved Quantum Differential Algorithm for Stochastic Flow Shop Scheduling Problem". In Proc. IEEE International Conference on Control and Automation 2009,pp.12351240.

[6] A. Draa , S. Meshoul, H. Talbi and M. Batouche, "A Quantum-Inspired Differential Evolution Algorithm for Solving the N-Queens Problem". The International Arab Journal of Information Technology,Vol. 7, No. 1, 2010 pp. 21-27.

[7] K.H. Han, J.H. Kim, "Quantum-inspired evolutionary algorithm for a class of combinatorial optimization," IEEE Trans. Evolut. Comput.,6(6) (2002) 580-593.

[8] Javidi and Saeed Mehrabi, "On Evolutionary Algorithms for Maximum Independent Set Problem" Journal of
Artificial Intelligence: Theory and Application (Vol.12010/Iss.2), pp.54-59.

[9] Giandomenico, M, et al. "A New Approach to the Stable Set Problem Based on Ellipsoids," Integer Programming andCombinatorial Optimization, Springer 2011, pp. 223234.

[10] PM Pardalos, OA Prokopyev, S Busygin "continuous approaches for solving discrete optimization problems." Handbook on modelling for discrete optimization, 2006 Springer

[11] http://www.mathworks.com

[12] Grötzsch H., "Ein Dreifarbensatz für dreikreisfreie Netz auf der Kugel,” Z. Martin-Luther-Univ., 1958.

[13] Herschel A.S., Sir Wm., "Hamilton's Icosian Game," Quart. J. Pure Applied Math.

[14] Petersen J., Die Theorie der regulären Graphen, Acta Math., 1891.

[15] Ramsey F.P. , On a problem of formal logic, Proc. London Math. Soc., 1930. 